\title{
Otitis Externa in Secondary Care: A Change in Our Practice Following a Full Cycle Audit
}

\author{
Zhaobo Liu ${ }^{1}$ Mohd Afiq Mohd Slim ${ }^{1}$ Catherine Scally ${ }^{1}$ \\ ${ }^{1}$ Department of Otolaryngology, Northern Health and Social Care \\ Trust, Antrim, United Kingdom of Great Britain and Northern Ireland \\ Int Arch Otorhinolaryngol 2018;22:250-252. \\ Address for correspondence Zhaobo Liu, MBChB, BMedSci, \\ Department of Otolaryngology, Northern Health and Social Care \\ Trust, 45 Bush Road, Antrim BT42 1WR, United Kingdom of Great \\ Britain and Northern Ireland (e-mail: paulliuzb@doctors.org.uk).
}

\begin{abstract}
Introduction Patients presenting with otitis externa are a common thing in otolaryngology units. However, the practice has not been standardized due to a lack of consensus over the management of this condition in secondary care. The National Institute for Health and Care Excellence (NICE) guideline has been published targeting the general practitioners, but it may be relevant in cases of hospital first-time attenders.

Objective To conduct an audit of the investigative and prescription practice for hospital first-time attenders in our department against the NICE guideline for otitis externa.

Methods The case notes of the patients presenting with otitis externa were reviewed. The data collation included the performance of ear swabs and choice of eardrops.

Results An initial audit showed that ear swabs were sent in 14 out of 19 cases, of which 11 grew either Pseudomonas aeruginosa or Staphylococcus aureus (organisms that are sensitive to empirical treatment). A re-audit showed higher adherence to NICE recommendations, with ear swabs sent in only 3 out of 25 cases. The initial audit also demonstrated Sofradex (Sanofi-Aventis, Paris, France) as the most popular empirical eardrop. Following our recommendation, the re-audit showed that Betnesol-N (GSK, Brentford, UK) was administered in 24 out of 25 cases.

Keywords

- otitis externa

- microbiology

- epidemiology Conclusion We recommend Betnesol-N due to its cost-effectiveness. Ear swabs should be reserved for refractory cases only. Posters and email reminders are effective means of disseminating information within the hospital.
\end{abstract}

\section{Introduction}

Otitis externa is a common condition affecting $1-3 \%$ of the population. ${ }^{1}$ The patients often present initially to primary care. They should be referred to an ear, nose and throat (ENT) specialist if the symptoms persist despite the initial treatment with antimicrobial drops. Multifactorial issues exist as to why this occurs. However, the most common reason to seek specialist input is to address the stenosed external acoustic meatus secondary to the edema and debris in the ear canal requiring microsuction, or if there is any suspicion of malignant otitis externa.

The National Institute for Health and Care Excellence (NICE) has issued a guideline for the investigation and treatment of otitis externa. ${ }^{2}$ According to the guideline, ${ }^{2}$ routine ear swabs should not be taken from patients presenting with otitis externa. This is because most of the empirical antimicrobial eardrops are sensitive to the usual pathogens. ${ }^{3}$ While there is a plethora of antimicrobial eardrops available, concerns arose that different preparations of eardrops were prescribed without considering the cost. Furthermore, the interpretation of culture results is made difficult by the fact that the reported bacterial susceptibility is determined for systemic antibiotics and not for topical administration., ${ }^{2,3}$ Concurrently, it is also difficult to differentiate the disease-causing organisms from the contaminants. ${ }^{3}$ However, ear swabs do have value in determining antibiotic resistant cultures in refractory cases (when the symptoms have lasted for more than 3 months). ${ }^{3}$ received

May 18, 2017

accepted

August 18, 2017

published online

September 19, 2017
DOI https://doi.org/

10.1055/s-0037-1606621. ISSN $1809-9777$.
Copyright (e 2018 by Thieme Revinter

Publicações Ltda, Rio de Janeiro, Brazil
License terms

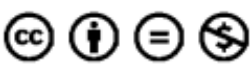


The clinical practices differ for first-time attenders with otitis externa in the secondary care setting. While some ENT departments would routinely perform ear swabs for these patients, as these cases were considered refractory on the basis that the initial treatment prescribed by the general practitioners (GPs) was not effective, other departments would disagree and reserve ear swabs for repeat attenders only. The cost of each ear swab with sensitivity culture is $£ 15$ in our unit. Considering the epidemiology of the condition, redundant ear swabs could cost the National Health Service (NHS) a significant amount of money per year. Therefore, a consensus needs to be reached regarding the practice of performing ear swabs routinely in first-time attenders with otitis externa to ENT specialist clinics.

In view of the aforementioned issues, the authors decided to conduct an audit to assess the current management of otitis externa in the ENT unit from the secondary care perspective.

\section{Methods}

We performed an initial audit of our practice in the ENT treatment room of our hospital from July to October 2016. The patients were selected based on the diagnosis of otitis externa recorded in the ward attenders' diary. Only first-time attenders with otitis externa were included. The medical notes of these patients were then assessed, and details pertaining to demographics, whether the ear swabs were taken or not upon presentation, type of eardrop prescribed, and the sensitivity of the ear swab cultures were all collated. The initial cycle results were than compared with the NICE guideline ${ }^{2}$ recommendations. Subsequently, new recommendations based on the findings were disseminated in the form of email reminders and a poster in the ENT treatment room. A re-audit of the practice was performed for the period of December 2016 to May 2017 using the same patient selection criteria as our initial audit to assess for adherence to the new recommendations. The data was collated and analyzed using the Microsoft Excel (Microsoft Corp., Redmond WA, US) software.

\section{Results}

A total of 19 patients ( 9 male and 10 female) with a mean age of 37 years were included in the initial audit. Of these 19 patients, 14 had ear swabs taken (-Fig. 1). Of the ear swabs cultured, only 1 case was resistant to aminoglycoside-based antibiotic drops. Sofradex (Sanofi-Aventis, Paris, France) was the most commonly prescribed eardrop (-Fig. 2). One patient received a combination of Sofradex and Canesten (Bayer, Leverkusen, Germany) eardrops.

In the second cycle of the audit period, 25 patients ( 9 male and 16 female) with a mean age of 46 years were assessed following the improvement intervention (email reminder and poster in the treatment room). Only 3 ear swabs were taken (none resistant to aminoglycoside-based antibiotic drops). Betnesol-N (GSK, Brentford, UK) was prescribed to 24 patients, and the Otomize (Teva UK Ltd, Essex, UK) ear spray was prescribed to 1 patient.

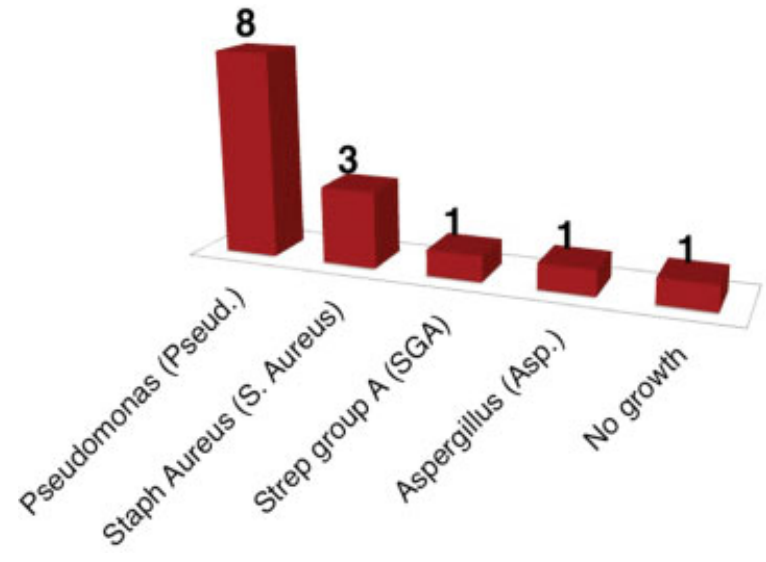

Fig. 1 Organisms cultured from ear swabs (initial audit).

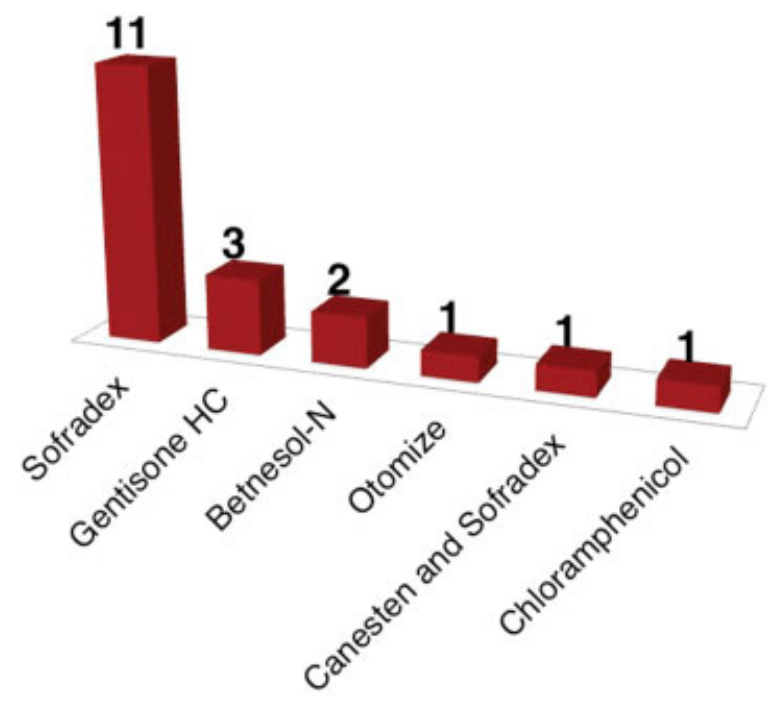

Fig. 2 Choice of eardrop (initial audit).

\section{Discussion}

While all patients presenting to the ENT treatment room had been previously treated by their GP, ear swabs should not be routinely taken from first-time attenders to the ENT specialist clinic. It is evident from - Fig. 1 that ear swab cultures reflect culture results from the community, with a predominance of Pseudomonas aeruginosa and Staphylococcus aureus. ${ }^{4}$ Furthermore, antibiotic resistance based on the ear swab culture was low, with only 1 case of resistance to the aminoglycoside group from 14 cultures.

It is useful to note that the patients presenting to secondary care could have been treated in primary care by a variety of management strategies. A survey of GPs in 2011 showed that $42 \%$ of patients received no treatment by their GP prior to referral to the ENT clinic, while $44 \%$ were prescribed only oral antibiotics, or oral antibiotics combined with topical antibiotics, and $14 \%$ were prescribed topical antibiotics alone. ${ }^{5}$ The effectiveness of oral antibiotics against otitis externa is questionable, as they achieve a much lower concentration in an area, ${ }^{6,7}$ while the fear of the potential ototoxic side effects of 
Table 1 Antimicrobial eardrops' sensitivity, including cost

\begin{tabular}{|l|l|l|l|l|l|}
\hline & Pseud. & S. aureus & SGA & Asp. & $\begin{array}{l}\text { Cost/ } \\
10 \mathrm{~mL}\end{array}$ \\
\hline Sofradex & Sensitive & Sensitive & Sensitive & Resistant & $£ 7.50^{*}$ \\
\hline $\begin{array}{l}\text { Gentisone } \\
\text { HC }\end{array}$ & Sensitive & Sensitive & Sensitive & Resistant & $£ 4.76^{*}$ \\
\hline Betnesol-N & Sensitive & Sensitive & Sensitive & Resistant & $£ 2.39^{*}$ \\
\hline Otomize & Sensitive & Sensitive & Sensitive & Resistant & $£ 6.54^{*}$ \\
\hline
\end{tabular}

Abbreviations: Asp., Aspergillus fumigatus; Pseudo., Pseudomonas aeruginosa; S. aureus, Staphylococcus aureus; SGA, Streptococcus Group A. Note: ${ }^{*}$ Cost calculations. ${ }^{7}$

aminoglycoside-based topical preparations has limited their use in primary care. This is despite the fact that the empirical treatment for otitis externa in primary care includes topical antiseptics ( $2 \%$ acetic acid, boric acid) and aminoglycoside eardrops as first-line and second-line respectively. ${ }^{3}$

It is likely that patients with otitis externa presenting to secondary care are usually patients whose treatment was not optimized in primary care, require microsuction or wick insertion to improve the eardrop delivery rather than cases of resistance to the empirical treatment. Based on this, ear swabs should not be performed in the ENT treatment room without a trial of empirical treatment first by the ENT specialist. Oral antibiotics will have a role in the complicated management of otitis externa (pinna cellulitis or malignant otitis externa).

When a second-line treatment needs to be considered by the ENT specialist following the initial management in primary care with antiseptics, Betnesol- $\mathrm{N}$ is the most costeffective antimicrobial eardrop ( Table 1 ). ${ }^{3,8}$ This can be appreciated from our cohort of ear swabs sensitivity results, as it has shown to have the same antimicrobial effectiveness as the other aminoglycoside-based eardrops. Besides aminoglycoside-based eardrops, some also consider the unlicensed use of ciprofloxacin-based eardrops. ${ }^{3}$ However, evidence comparing the effectiveness of these two eardrops is lacking; therefore Betnesol- $\mathrm{N}$ is the most reasonable choice for the empirical treatment of otitis externa due to its cost and antimicrobial sensitivity.

This full cycle audit used the display of posters at the point of care (ENT treatment room) and reminder emails to promote our recommendation for changes in practice. Email reminders have been shown previously to demonstrate a statistically significant difference favoring the email intervention over no intervention for compliance with clinical practice guidelines. ${ }^{9}$ However, computer reminders at the point of care were shown to have a small impact on guideline adherence. ${ }^{10}$ In fact, it has been suggested that reminders will only be effective if the cause of the poor performance was not having the information at the point of care. ${ }^{11}$ This audit has demonstrated that the combination of electronic and poster reminders helps improve the patients' care.

\section{Conclusion}

Ear swabs should not be routinely performed on first-time attenders to the ENT specialist clinics in secondary care. They should only be considered after a failed trial of empirical treatments or in patients presenting with chronic ear infections. We recommend Betnesol- $\mathrm{N}$ as an empirical secondline choice due to its cost-effectiveness in the treatment of otitis externa when in secondary care. This audit provides an example of how service at an ENT outpatient clinic could be improved after disseminating clinical guidance through email and poster reminders. Given that this is a very common condition, these recommendations could lead to significant cost reductions if applied to ENT departments across the country.

\section{Financial Support}

This work did not receive any financial support.

\section{References}

1 Wipperman J. Otitis externa. Prim Care 2014;41(01):1-9

2 National Institute for Health and Care Excellence (NICE). Clinical Knowledge Summaries: Otitis externa. Available from: https:// cks.nice.org.uk/ovtitis-externa\#! topicsummary. [Accessed $1^{\text {st }}$ February 2017]

3 Llor C, McNulty CAM, Butler CC. Ordering and interpreting ear swabs in otitis externa. BMJ 2014;349:g5259. Doi: 10.1136/bmj.g5259

4 Roland PS, Stroman DW. Microbiology of acute otitis externa. Laryngoscope 2002;112(7 Pt 1):1166-1177

5 Pabla L, Jindal M, Latif K. The management of otitis externa in UK general practice. Eur Arch Otorhinolaryngol 2012;269(03):753-756

6 Rosenfeld RM, Schwartz SR, Cannon CR, et al. Clinical practice guideline: acute otitis externa. Otolaryngol Head Neck Surg 2014; 150(Suppl 1):S1-S24

7 Kaushik V, Malik T, Saeed SR. Interventions for acute otitis externa. Cochrane Database Syst Rev 2010;(01):CD004740

8 Joint Formulary Committee. British National Formulary. 72 ed. London: BMJ Group and Pharmaceutical Press; 2016

9 Lobach DF. Electronically distributed, computer-generated, individualized feedback enhances the use of a computerized practice guideline. Proceedings of the AMIA Annual Fall Symposium. 1996:493-7

10 Shojania KG, Jennings A, Mayhew A, Ramsay C, Eccles M, Grimshaw J. Effect of point-of-care computer reminders on physician behaviour: a systematic review. CMAJ 2010;182(05):E216-E225

11 Moulding NT, Silagy CA, Weller DP. A framework for effective management of change in clinical practice: dissemination and implementation of clinical practice guidelines. Qual Health Care 1999;8(03):177-183 